\title{
Geometry on nodal curves
}

\author{
Ziv Ran
}

\begin{abstract}
Given a family $X / B$ of nodal curves we construct canonically and compatibly with basechange, via an explicit blow-up of the Cartesian product $X^{r} / B$, a family $W^{r}(X / B)$ that we show is isomorphic to the relative flag Hilbert scheme parametrizing flags of subschemes of fibres of $X / B$ with colengths $1, \ldots, r$. Although $W^{r}(X / B)$ is singular, the important sheaves on it are locally free, which allows us to study some intersection theory on it and deduce enumerative applications, including some relative multiple point formulae enumerating the length- $r$ schemes contained simultaneously in some fibre of $X / B$ and some fibre of a given map from $X$ to a smooth variety.
\end{abstract}

\section{Introduction}

One of the important facts which make geometry, in particular enumerative geometry, on a smooth curve $X$ relatively simple is the existence of simple and quite tractable parameter spaces for subschemes of $X$ of given length $r$, be it the symmetric product $\operatorname{Sym}^{r}(X)$, which in fact is isomorphic to the Hilbert scheme $\operatorname{Hilb}^{r}(X)$, or the Cartesian product $X^{r}$, which parameterizes subschemes in an $(r !: 1)$ fashion and is sufficient for many applications, especially enumerative ones. One might say that what is essential about these parameter spaces is that they are enumerative-geometric, i.e.

(i) geometric, which effectively means they admit a morphism to the Hilbert scheme, through which natural geometric loci may be defined by pullback;

(ii) enumerative, meaning that the relevant cohomology classes and intersection numbers that may enter into the enumeration of those natural geometric loci are computable.

Mutatis mutandis, similar considerations also hold for families of smooth curves. In [Ran85], the author studied from this viewpoint enumerative projective geometry for families of smooth curves, obtaining, inter alia, a general relative multiple point formula. This is a formula enumerating the length- $r$ subschemes of the fibres of a given family $X / B$ whose image under a given map

$$
f: X \rightarrow Y
$$

is a single reduced point.

Incongruously, it seems these ideas and results have yet to be extended to singular curves and families of such. This paper is a step in that direction in the case of nodal curves, i.e. curves with only ordinary double points as singularities. To a family

$$
\pi: X \rightarrow B
$$

of nodal curves and a natural number $r$ we shall associate canonically a family

$$
\pi_{r}: W^{r}(X / B) \rightarrow B
$$

Received 16 January 2004, accepted in final form 16 November 2004, published online 1 September 2005.

2000 Mathematics Subject Classification 14N10, 14H10, $14 \mathrm{C} 05$.

Keywords: nodal curve, family of curves, subscheme, enumerative geometry, multiple points, projective geometry.

The author was partially supported by NSA grant MDS904-02-1-0094; reproduction and distribution of reprints by US government permitted.

This journal is (C) Foundation Compositio Mathematica 2005. 


\section{Z. RAN}

which is enumerative-geometric in the above sense and has a number of other favorable properties. $W^{r}(X / B)$ is functorial in $B$, i.e. its formation commutes with base-change; in fact, $W^{r}(X / B)$ is a canonical and explicit blow-up of the Cartesian fibre product $X^{r} / B$ (more explicitly and directly, of $\left.W^{r-1}(X / B) \times_{B} X\right)$ in a suitable sheaf of ideals. We will show that $W^{r}(X / B)$ admits a morphism to the relative Hilbert scheme $\operatorname{Hilb}^{r}(X / B)$. In fact, we will subsequently show that $W^{r}(X / B)$ is isomorphic to the relative flag-Hilbert scheme, which parametrizes chains

$$
z_{1} \subset z_{2} \cdots \subset z_{r}
$$

where each $z_{i}$ is a length- $i$ subscheme of a fibre of $X / B$. The relatively simple relationship of $W^{r}(X / B)$ and $W^{r-1}(X / B)$, in the form of the natural blow-up map

$$
b_{r}: W^{r}(X / B) \rightarrow W^{r-1}(X / B) \times_{B} X,
$$

makes these spaces more amenable to enumerative computations than the Hilbert scheme itself.

The fact that $W^{r}(X / B)$ admits a morphism to Hilb implies that, for any vector bundle $L$ on $X$, $W^{r}(X / B)$ carries a 'tautological' bundle (also called a secant bundle) $S^{r}(L)$, whose fibre at a point is the restriction of $L$ on the corresponding scheme. We will see that due to the simple relationship of $W^{r}(X / B)$ and $W^{r-1}(X / B), S^{r}(L)$ can be analyzed conveniently with exact sequences that relate it to $S^{r-1}(L)$. This fact, together with the fact that certain 'diagonal' divisors become Cartier on $W^{r}(X / B)$, enables us to consider some intersection theory on these spaces and apply it to enumerative questions (although the complete intersection theory of the $W^{r}(X / B)$ is yet to be worked out).

We note that the $W^{r}(X / B)$, as total spaces, are always singular for $r \geqslant 3$, as soon as the family $X / B$ has singular fibres. However, when $B$ is smooth, $W^{r}(X / B)$ is normal and Cohen-Macaulay (cf. Proposition 2.3). In enumerative applications, at least those developed so far, the singular nature of the $W^{r}(X / B)$ is irrelevant; what matters is that they are enumerative-geometric in the sense described above.

The remainder of this paper is largely devoted to multiple-point formulae. In the case of a map $f$ to a Grassmannian, one can define and enumerate a multiple-point scheme $M_{r}(f)$ as a more-orless direct consequence of the existence of a tautological bundle on $W^{r}(X / B)$ (whose fibre at a point is the space of functions, or sections of a vector bundle, on the corresponding subscheme), and its relation to the tautological bundles on the Grassmannian. This result can be extended to the case of a target space which is 'pseudo-Grassmannian' in the sense that its diagonal admits a nice global description as the zero-scheme of a vector bundle. In the case of a map to a general (smooth) variety $Y$, multiple-point schemes $M_{r}(f)$ can still be defined via an embedding of $Y$ in a pseudo-Grassmannian (e.g. Projective space), and then enumerated by applying a residualintersection formula. It can, in fact, be shown that $M_{r}(f)$ can be defined intrinsically, independently of the pseudo-Grassmannian embedding (hence even for non-projective or non-algebraic $Y$ ), but that argument is disproportionately involved considering the paucity of applications and is not presented here. The version we do present follows a suggestion by the referee.

Perhaps the main question raised and left open by this paper is the full computation of the subring of the cohomology (or Chow) ring of the $W^{r}(X / B)$ generated by the diagonal divisor classes $\left[\Delta^{k}\right], k=2, \ldots, r$, corresponding to the loci where the $k$ th point coincides with one of the preceding points. This would allow the explicit evaluation of all the multiple-point loci that we compute, beyond the few fragmentary results given here (generally for $r \leqslant 3$ ). However, see [Ran05b, Ran04b] for some recent progress.

The paper is organized as follows. The construction of the parameter spaces $W^{r}(X / B)$ is begun in $\S 1$ where the case $r \leqslant 3$ is considered in explicit detail. The general case is completed in $\S 2$ by an inductive construction, and the relation with the Hilbert scheme is worked out in full. 


\section{GeOMETRY ON NODAL CURVES}

In $\S 3$ we give enumerative applications, including several multiple-point formulae for maps to targets of various degrees of generality, and some concrete examples, mainly for maps to $\mathbb{P}^{2}$ and $\mathbb{P}^{3}$.

This paper is, in part, a continuation of [Ran04a], where more particular enumerative results were obtained for $r=2$, in effect by an $a d$ hoc version of some of the methods of this paper. We will work over $\mathbb{C}$. However, we do not see any significant obstruction to extending the results over an algebraically closed field of arbitrary characteristic.

\section{Parameter spaces for pairs and triples}

Let

$$
\pi: X \rightarrow B
$$

be a flat family of curves over an irreducible variety, with all fibres nodal, i.e. each fibre of $\pi$ has at worst ordinary double points as singularities. Typically, the generic fibre will be smooth, but we do not assume this. Our purpose is to construct a natural and explicit birational modification $W^{r}(X / B)$ of the relative Cartesian product $X^{r} / B$, which will serve as our basic 'configuration space' on which to do enumerative geometry. In this section we begin by considering in explicit detail the case of $r \leqslant 3$. Subsequently, the construction will proceed by induction.

First, we obviously set

$$
W^{1}(X / B)=X / B
$$

Next we consider the easy but significant case $r=2$. Note that, at a critical point $p$ of $\pi$ (i.e. a singular point of a fibre $X_{b}=\pi^{-1}(b)$ ), our family is formally equivalent to a subvariety of $\mathbb{A}^{2} \times B$ given by

$$
x y=a
$$

where $x, y$ are coordinates on $\mathbb{A}^{2}$ and $a$ is an element of $\hat{\mathfrak{m}}_{B, b}$, which may also be viewed as a (formal) mapping of $B$ to the base $\left(=\mathbb{A}^{1}\right)$ of the versal deformation of a node, pulling back the germ at $p$ of $X / B$. The analogous statements obtained by replacing 'formal' by 'complex analytic' also hold, and it is basically a matter of taste whether one uses the formal or analytic setting. Note that $X$ is smooth at $p$ if and only if $a \notin \hat{\mathfrak{m}}_{B, b}^{2}$. If $B$ is a smooth curve, $a$ is either zero or may be taken to be of the form $a=t^{k}$, where $t$ is a local parameter on $B$ and $k \geqslant 1$, and $k=1$ if and only if $X$ is smooth at $p$. The construction we shall undertake will be manifestly local about critical points such as $p$, as well as compatible with base-change; therefore, for many purposes it will suffice to consider the 'universal' case $x y=t$.

Now consider the fibre square $X^{2} / B$ and let $D \subset X^{2} / B$ be the diagonal. Note that $D$ is a Cartier divisor at all points $(p, p)$ such that $\pi$ is smooth (i.e. submersive) at $p$. On the other hand, at points $(p, p)$ where $p$ is a critical point, i.e. a fibre singularity, of $\pi, X^{2} / B$ is given formally or analytically by

$$
x_{1} y_{1}=x_{2} y_{2}=a,
$$

and $D$ is given by the two equations

$$
x_{1}=x_{2}, y_{1}=y_{2}
$$

moreover, if the total space $X$ is smooth, then $X^{2} / B$ is smooth (over $\mathbb{C}$ ) except at those points $(p, p)$. We let

$$
b_{2}: W^{2}(X / B) \rightarrow X^{2} / B
$$

denote the blow-up of $D \subset X^{2} / B$. As $D$ is Cartier away from the singular points $(p, p)$ as above, the blow-up coincides with the blow-up locally at those points of the ideal $\left(x_{1}-x_{2}, y_{1}-y_{2}\right)$, cf. (1.3). 


\section{Z. RAN}

Let

$$
\Delta^{2} \subset W^{2}(X / B)
$$

be the exceptional divisor, defined by

$$
\mathcal{I}_{\Delta^{2}}=b_{2}^{*}\left(\mathcal{I}_{D}\right)
$$

(this is not to be confused with the exceptional locus, i.e. the locus of all points of $W^{2}(X / B)$ where $b_{2}$ is not an isomorphism locally). Note that the construction of $\Delta^{2} \subset W^{2}(X / B)$ is manifestly canonical and compatible with base-change. To analyze it, it will suffice to consider the situation locally along the exceptional locus of $b_{2}$ where we may use formal or analytic coordinates as above.

In terms of these coordinates, over a neighborhood (formal or analytic) of each $(p, p), W^{2}(X / B)$ is covered by two open affines denoted by $U_{x, p}^{2}, U_{y, p}^{2}$ (or just by $U_{x}^{2}, U_{y}^{2}$ if $p$ is understood). The coordinate ring of $U_{x, p}^{2}$ is generated over that of $X^{2} / B$ by a symbol $\left[\left(y_{2}-y_{1}\right) / /\left(x_{2}-x_{1}\right)\right]$ subject to the evident relation

$$
\left(x_{2}-x_{1}\right)\left[\left(y_{2}-y_{1}\right) / /\left(x_{2}-x_{1}\right)\right]=y_{2}-y_{1} .
$$

Note that

$$
x_{1}\left(x_{2}-x_{1}\right)\left[\left(y_{2}-y_{1}\right) / /\left(x_{2}-x_{1}\right)\right]=x_{1}\left(y_{2}-y_{1}\right)=y_{2}\left(x_{1}-x_{2}\right),
$$

hence we may, and shall, write $\left[\left(y_{2}-y_{1}\right) / /\left(x_{2}-x_{1}\right)\right]$ as $-\left[y_{2} / / x_{1}\right]$; similarly, the same may also be written as $-\left[y_{1} / / x_{2}\right]$, and therefore when the index range is understood we may write $-[y / / x]$. Similar comments apply over the other open $U_{y, p}^{2}$ where a generator $\left[x_{1} / / y_{2}\right]=\left[x_{2} / / y_{1}\right]=[x / / y]$ is defined and, of course, on the overlap $U_{x, p}^{2} \cap U_{y, p}^{2}$ we have

$$
\left[y_{2} / / x_{1}\right]\left[x_{1} / / y_{2}\right]=1
$$

Henceforth, we shall denote $U_{x, p}^{2}, U_{y, p}^{2}$ as $U([y / / x]), U([x / / y])$, respectively.

Thus the exceptional locus of $b_{2}$ consists of a $\mathbb{P}^{1}$ over each point $(p, p)$ as above. Moreover, it is easy to see that if $X$ is smooth, then so is $W^{2}(X / B)$ : indeed in $U([y / / x])$ (respectively $U([x / / y])$ ), a set of coordinates (or a regular system of parameters) is given by $x_{1}, x_{2}, y_{1}$ (respectively $x_{2}, y_{1}, y_{2}$ ). In fact, if $X$ is a smooth surface then, by $(1.2),(p, p)$ is just a 3-fold ordinary double point and $b_{2}$ is one of its two small resolutions. As an aside, it is curious to note that this resolution may be obtained determinantally, i.e. via (1.2) we obtain locally a mapping

$$
\begin{gathered}
M: X^{2} / B \rightarrow M_{2 \times 2}^{1}, \\
M=\left(\begin{array}{ll}
x_{1} & x_{2} \\
y_{2} & y_{1}
\end{array}\right),
\end{gathered}
$$

to the space of $(2 \times 2)$ matrices of rank $\leqslant 1$ and $W^{2}(X / B)$ is just obtained by taking the fibre product of $X^{2} / B$ via $M$ with what is known as the 'canonical determinantal resolution' of $M_{2 \times 2}^{1}$, defined by

$$
R_{2 \times 2}^{1}=\left\{(A, B) \in M_{2 \times 2}^{1} \times \mathbb{P}^{1}: B A=0\right\} .
$$

Next, we claim that the natural rational map of $X^{2} / B$ to the Hilbert scheme $\operatorname{Hilb}_{2}(X / B)$, assigning a pair $p \neq q$ to the ideal $\mathcal{I}_{p, q}<\mathcal{O}_{X}$, lifts to a morphism, i.e. a regular map

$$
J_{2}: W^{2}(X / B) \rightarrow \operatorname{Hilb}_{2}(X / B) .
$$

$A$ priori, $J_{2}$ is just a rational map, and as such it is clearly compatible with the respective natural maps of $W^{2}(X / B)$ and $\operatorname{Hilb}_{2}(X / B)$ to $\operatorname{Sym}^{2}(X / B)$ (see [Ang81] for a general construction of the map from the Hilbert scheme to the Chow variety). By the projectivity of blow-up morphisms and of Hilbert schemes, respectively, these maps are proper (even projective); hence by the GAGA principle it would suffice to check that $J_{2}$ exists as a holomorphic map (see Remark 1.1 below for a formal analogue, in arbitrary characteristic, of this argument). Clearly it suffices to check the holomorphic 


\section{GeOMETRY ON NODAL CURVES}

nature of $J_{2}$ locally along the exceptional locus. To this end, it suffices finally to note that, in the open subset $U_{x, p}^{2}, J_{2}$ is given explicitly by sending a point with coordinates $\left(x_{1}, x_{2},\left[y_{2} / / x_{1}\right]\right)$ to the ideal

$$
J_{2}\left(x_{1}, x_{2},\left[y_{2} / / x_{1}\right]\right)=\left(x y-a,\left(x-x_{1}\right)\left(x-x_{2}\right), y-y_{1}+\left[y_{2} / / x_{1}\right]\left(x-x_{1}\right)\right)
$$

where, of course, we set

$$
y_{1}=x_{2}\left[y_{1} / / x_{2}\right], y_{2}=x_{1}\left[y_{2} / / x_{1}\right], a=x_{1} y_{1}=x_{2} y_{2}=x_{1} x_{2}\left[y_{2} / / x_{1}\right] .
$$

Indeed, it is elementary that the right-hand side of (1.4) defines an ideal of colength 2 whose cosupport contains $\left(x_{1}, y_{1}\right),\left(x_{2}, y_{2}\right)$, and therefore this right-hand side defines a morphism to Hilb that coincides with $J_{2}$ generically (e.g. whenever $\left.\left(x_{1}, y_{1}\right) \neq\left(x_{2}, y_{2}\right)\right)$. This implies our assertion. The case of $U_{y, p}^{2}$ is similar.

Remark 1.1. The use of holomorphic coordinates and the GAGA principle to show the regularity of $J_{2}$ can be circumvented and replaced by formal coordinates, meaningful in characteristic $p$, as follows. First, it is well known and easy to prove that any nodal curve, embedded in $\mathbb{P}^{N}$ by a complete linear system of sufficiently high degree on each component, yields a smooth (unobstructed) point of the Hilbert scheme of $\mathbb{P}^{N}$, and consequently any family of nodal curves is obtained by pullback from a family with smooth base. Since $J_{2}$ (as rational map), its source and target are all base-change compatible, it suffices to check the regularity of $J_{2}$ in the case of a smooth base $B$. If $B$ is smooth then $W^{2}(X / B)$ is normal by Corollary 2.2.1 below. On the other hand, formal-analytic considerations as above show that $J_{2}$ exists as a continuous, formally regular map, in the sense that for any point $w$ in $W=W^{2}(X / B)$ there corresponds a well-defined point $h=J_{2}(w) \in H=\operatorname{Hilb}_{2}(X / B)$ and

$$
J_{2}^{*}\left(\hat{\mathcal{O}}_{H, h}\right) \subseteq \hat{\mathcal{O}}_{W, w},
$$

where $\hat{\mathcal{O}}$ denotes formal completion. Then, for any $g \in \mathcal{O}_{H, h}, J_{2}^{*}(g)$ is a rational function on $W$ that is also in $\hat{\mathcal{O}}_{W, w}$, and by a well-known property of normal varieties (cf. [Mum88, ch. 3, § 9]) it follows that $J_{2}^{*}(g) \in \mathcal{O}_{H, h}$. Hence $J_{2}$ is a morphism.

Yet another approach to proving the regularity of $J_{2}$, suggested by an anonymous correspondent, is to note that $J_{2}$ corresponds to the subscheme $\Gamma^{3} \subset W^{2} \times_{B} X$ as in (1.5) below and the regularity of $J_{2}$ is equivalent to the flatness of $\Gamma^{3}$ over $W^{2}$. By the formal criterion for flatness, this flatness may be checked by passing to formal completions where we have the formal coordinates $x_{i}, y_{j}$ as above, and the previous computations as in (1.4) prove flatness.

Now, we study in detail the case $r=3$. Let

$$
\Gamma^{3} \subset W^{2} \times_{B} X
$$

denote the pullback of the tautological subscheme of $\operatorname{Hilb}_{2}(X / B)$ via the map $J_{2}$. Note the schemetheoretic equality

$$
\Gamma^{3}=\Gamma_{1}^{3} \cup \Gamma_{2}^{3}
$$

where

$$
\Gamma_{i}^{3} \subset W^{2} \times_{B} X
$$

is the graph of the natural projection $W^{2} \rightarrow X$ : indeed this follows easily from the facts that the left-hand side of (1.5) is flat over $W^{2}$ and contains the right-hand side, the right-hand side is reduced, and both sides agree generically over $W^{2}$. In particular, we see that $\Gamma^{3}$ is reduced. We define

$$
W^{3}=W^{3}(X / B):=B_{\Gamma^{3}}\left(W^{2}(X / B) \times_{B} X\right),
$$

i.e. the blow-up of $W^{2} \times_{B} X$ in $\Gamma^{3}$, with natural map

$$
b_{3}: W^{3} \rightarrow W^{2} \times_{B} X .
$$




\section{Z. RAN}

Clearly $W^{3}$ is reduced. Let $\Delta^{3} \subset W^{3}$ be the natural exceptional (Cartier) divisor supported on $b_{3}^{-1}\left(\Gamma^{3}\right)$, with ideal

$$
\mathcal{I}_{\Delta^{3}}=b_{3}^{*}\left(\mathcal{I}_{\Gamma^{3}}\right)
$$

and $\Delta_{i}^{3}=b_{3}^{-1}\left(\Gamma_{i}^{3}\right), i=1,2$, its components (as Weil, in fact non-Cartier divisors). From the computations below it will follow that all these divisors are reduced.

To analyze this construction, we work over $U([y / / x])$. There, note that the expression $\left(y_{3}-y_{1}\right)$ $\left(x_{3}-x_{2}\right)$ viewed, for example, as a function on

$$
V:=U([y / / x]) \times{ }_{B} U
$$

is divisible by $x_{2}$; indeed, setting

$$
R=y_{2}-[y / / x] x_{3}-y_{3}+y_{1},
$$

it is easy to check that $x_{2} R=\left(y_{3}-y_{1}\right)\left(x_{3}-x_{2}\right)$. We claim next that the ideal of $\Gamma^{3}$ in $V$ is generated by $\left(x_{3}-x_{1}\right)\left(x_{3}-x_{2}\right)$ and $R$. Indeed, the subscheme $\Gamma^{\prime}$ defined by the latter projects isomorphically to the subscheme of the ' $x$-axis' defined by $\left(x_{3}-x_{1}\right)\left(x_{3}-x_{2}\right)$; hence, it is flat over $W^{2}$, and of course $\Gamma^{3}$ is also flat over $W^{2}$. Since $\Gamma^{3}$ and $\Gamma^{\prime}$ clearly coincide generically over $W^{2}$, they coincide, as claimed.

Thus we see that $b_{3}^{-1}(V)$ may be realized in the standard way as a subscheme of $V \times \mathbb{P}^{1}$ and as such is covered by the two standard opens pulled back from $\mathbb{P}^{1}$. One of these is the domain of regularity of the rational function

$$
-\left[y / / x^{2}\right]:=\frac{R}{\left(x_{3}-x_{1}\right)\left(x_{3}-x_{2}\right)},
$$

and it is easy to check that, as rational functions,

$$
\left[y / / x^{2}\right]=\frac{y_{i}}{x_{j} x_{k}}
$$

whenever $\{i, j, k\}=\{1,2,3\}$, justifying the notation; we denote this open by $U\left(\left[y / / x^{2}\right]\right)$. Note, trivially, that the regularity of $\left[y / / x^{2}\right]$ implies the regularity of $\left[y_{i} / / x_{j}\right], \forall i, j$, so $U\left(\left[y / / x^{2}\right]\right)$ indeed lies over $U([x / / y])$. On the other standard open of $V \times \mathbb{P}^{1}$ the function

$$
\left[x^{2} / / y\right]=\frac{x_{j} x_{k}}{y_{i}}
$$

is regular; however, its domain of regularity does not lie entirely over $U([y / / x]$ ) (nor, for that matter, entirely over $U([x / / y]))$.

Analogous comments apply to the part of $W^{3}$ over $U([x / / y])$ which gives rise to symbols $\left[x / / y^{2}\right],\left[y^{2} / / x\right]$. Setting

$$
U\left(\left[y^{2} / / x\right],\left[x^{2} / / y\right]\right):=U\left(\left[y^{2} / / x\right]\right) \cap U\left(\left[x^{2} / / y\right]\right)
$$

i.e. the common regularity domain of $\left[y^{2} / / x\right]$ and $\left[x^{2} / / y\right]$, note by construction that the regularity domains

$$
U\left(\left[y / / x^{2}\right]\right), \quad U\left(\left[y^{2} / / x\right],\left[x^{2} / / y\right]\right), \quad U\left(\left[x / / y^{2}\right]\right)
$$

already cover $W^{3}(X / B)$.

We claim next that the natural rational map $J_{3}$ of $W^{3}(X / B)$ to the relative Hilbert scheme $\operatorname{Hilb}_{3}(X / B)$ is a morphism. Using again the projectivity of $W^{3}(X / B)$ and $\operatorname{Hilb}_{3}(X / B)$ over $\operatorname{Sym}^{3}$ $(X / B)$ and the GAGA principle, it suffices to check that $J_{3}$ extends as a holomorphic map (the 'cycle map' $\operatorname{Hilb}_{3}(X / B) \rightarrow \operatorname{Sym}^{3}(X / B)$ is constructed in great generality by Angéniol [Ang81] or see [Kol96]); alternatively, one could argue as in Remark 1.1. The extension is a local assertion and is, moreover, either obvious or a consequence of the analogous result for $J_{2}$, except at the 


\section{GeOMETRY ON NODAL CURVES}

points of $W^{3}$ lying over $(p, p, p) \in X^{3} / B$, so it suffices to check it on opens

$$
U\left(\left[y / / x^{2}\right]\right), \quad U\left(\left[y^{2} / / x\right],\left[x^{2} / / y\right]\right), \quad U\left(\left[x / / y^{2}\right]\right)
$$

as above. Over $U\left(\left[y / / x^{2}\right]\right)$, it is easy to see that the expression

$$
\left(y-y_{1}\right)\left(x-x_{2}\right)\left(x-x_{3}\right),
$$

considered as a function on $W^{3} \times_{B} X$, i.e. taken modulo $x y-x_{1} y_{1}$, is divisible by $x_{2} x_{3}$. Explicitly, setting

$$
R=\left(\left[y_{2} / / x_{3}\right]-\left[y / / x^{2}\right]\right) x^{2}+\left(\left[y_{1} / / x_{3}\right]+\left[y_{1} / / x_{2}\right]\right) x+y-\left(y_{1}+y_{2}+y_{3}\right)
$$

(recall that $\left[y_{2} / / x_{3}\right]=x_{1}\left[y / / x^{2}\right]$ etc.), we have

$$
x_{2} x_{3} R \equiv\left(y-y_{1}\right)\left(x-x_{2}\right)\left(x-x_{3}\right) \quad \bmod x y-x_{1} y_{1} .
$$

Then, on $U\left(\left[y / / x^{2}\right]\right), J_{3}$ takes a point with coordinates $x_{i}, y_{j}$ to the ideal

$$
J_{3}=\left(\left(x-x_{1}\right)\left(x-x_{2}\right)\left(x-x_{3}\right), R, x y-x_{1} y_{1}\right) .
$$

Since the latter ideal evidently has colength 3 over $W^{3}$, this makes $J_{3}$ a morphism over $U\left(\left[y / / x^{2}\right]\right)$. The case of $U\left(\left[x / / y^{2}\right]\right)$ is similar. Over $U\left(\left[y^{2} / / x\right],\left[x^{2} / y\right]\right)$, it is elementary to check as above that, always modulo $x y-x_{1} y_{1},\left(y-y_{1}\right)\left(y-y_{2}\right)\left(x-x_{3}\right)$ is divisible by $x_{3}$ and $\left(x-x_{1}\right)\left(x-x_{2}\right)\left(y-y_{3}\right)$ is divisible by $y_{3}$, and the ideal

$$
J_{3}=\left(\frac{\left(y-y_{1}\right)\left(y-y_{2}\right)\left(x-x_{3}\right)}{x_{3}}, \frac{\left(x-x_{1}\right)\left(x-x_{2}\right)\left(y-y_{3}\right)}{y_{3}}, x y-x_{1} y_{1}\right)
$$

has colength 3 over $W^{3}$ and yields the map to Hilb (cf. [Ran05c]).

\section{Parameter spaces for $r$-tuples}

In this section we will give the construction of our parameter spaces $W^{r}(X / B)$ for general $r$, proceeding by induction. It is convenient to summarize the construction and its main properties as follows.

Theorem 2.1. Define

$$
b_{r}: W^{r}(X / B) \rightarrow W^{r-1}(X / B) \times_{B} X
$$

inductively as the blow-up of the canonical subscheme

$$
\Gamma^{r} \subset W^{r-1}(X / B) \times_{B} X
$$

corresponding to the morphism

$$
J_{r-1}: W^{r-1}(X / B) \rightarrow \operatorname{Hilb}_{r-1}(X / B),
$$

and let $\Delta^{r}=b_{r}^{-1}\left(\Gamma^{r}\right)$ be the exceptional divisor and

$$
w_{r}: W^{r}(X / B) \rightarrow X^{r} / B
$$

be the natural map. Then:

(i) $W^{r}(X / B)$ is reduced and is irreducible if $X$ is; $\Gamma^{r}$ is reduced and has $r-1$ irreducible components each isomorphic to $W^{r-1}$; as functor of the family $X / B, W^{r}(X / B)$ commutes with base-change;

(ii) the natural rational map

$$
J_{r}: W^{r}(X / B) \rightarrow \operatorname{Hilb}_{r}(X / B)
$$

is a morphism; 


\section{Z. RAN}

(iii) for each node $p$ of $X / B$, an analytic or formal neighborhood $N$ of $w_{r}^{-1}(p, \ldots, p)$ in $W^{r}=$ $W^{r}(X / B)$ carries meromorphic or formal-rational functions

$$
\left[y^{i} / / x^{r-i}\right]=\left[y^{I} / / x^{J}\right], \quad\left[x^{i} / / y^{r-i}\right]=\left[x^{I} / / y^{J}\right]
$$

where $I \subset[1, r]$ is any index set of cardinality $i$ and complement $J$, and the domains of regularity

$$
U\left(\left[y / / x^{r-1}\right]\right), \ldots, U\left(\left[y^{i} / / x^{r-i}\right],\left[x^{r-i+1} / / y^{i-1}\right]\right), \ldots, U\left(\left[x / / y^{r-1}\right]\right)
$$

form a covering of $N$;

(iv) setting

$$
P_{i}^{r}=\prod_{j=1}^{i}\left(y-y_{j}\right) \prod_{j=i+1}^{r}\left(x-x_{j}\right) \in \mathcal{O}_{W^{r}}[x, y] /\left(x y-x_{1} y_{1}\right),
$$

over $U\left(\left[y^{i} / / x^{r-i}\right]\right), P_{i}^{r}$ is divisible by $x_{i+1} \cdots x_{r}$ and over $U\left(\left[x^{r-i} / / y^{i}\right]\right), P_{i}^{r}$ is divisible by $y_{1} \cdots y_{i}$; over $U\left(\left[y^{i} / / x^{r-i}\right],\left[x^{r-i+1} / / y^{i-1}\right]\right)$, the map $J_{r}$ is given by

$$
J_{r}=\left(x y-x_{1} y_{1}, \frac{P_{i}^{r}}{x_{i+1} \cdots x_{r}}, \frac{P_{i-1}^{r}}{y_{1} \cdots y_{i-1}}\right) ;
$$

(v) $W^{r+1}$ is covered by open sets over each of which either

(a) $\left[y^{i} / / x^{r+1-i}\right]$ is regular and $P_{i-1}^{r}\left(x_{r+1}, y_{r+1}\right) /\left(y_{1} \cdots y_{i-1}\right)$ is an equation for $\Delta^{r+1}$ (called an ' $x$-based equation'); or

(b) $\left.\left[x^{r-i+1} / / y^{i}\right]\right)$ is regular and $P_{i}^{r}\left(x_{r+1}, y_{r+1}\right) /\left(x_{i+1} \cdots x_{r}\right)$ is an equation for $\Delta^{r+1}$ (called a ' $y$-based equation').

Proof. For $r \leqslant 3$ all the statements have already been proven, so we may assume they hold for $r-1$. First (i) is clear from the fact that $W^{r}$ is an iterated blow-up of the Cartesian product $X^{r} / B$, while $\Gamma^{r}$ coincides with the union of the graphs (over $B$ ) of the coordinate projections $p_{i}: W^{r-1} \rightarrow X$ (the proof is as in the $r=3$ case). For the rest, we may, as before, work over

$$
U\left(\left[y^{i} / / x^{r-1-i}\right],\left[x^{r-i} / / y^{i-1}\right]\right) \subset W^{r-1},
$$

so in a suitable open set in $W^{r-1} \times_{B} X$ the ideal of $\Gamma^{r}$ is generated by

$$
\frac{P_{i}^{r-1}}{x_{i+1} \cdots x_{r-1-i}}, \quad \frac{P_{i-1}^{r-1}}{y_{1} \cdots y_{i-1}}
$$

(where we plug in $\left(x_{r}, y_{r}\right)$ for $\left.(x, y)\right)$. Thus the blow-up (in the part under consideration) is covered by two standard opens. In the first we have the regular function

$$
\frac{P_{i}^{r-1}}{x_{i+1} \cdots x_{r-1-i}} / \frac{P_{i-1}^{r-1}}{y_{1} \cdots y_{i-1}}=\frac{y_{r}-y_{i}}{x_{r}-x_{i}} \frac{y_{1} \cdots y_{i-1}}{x_{i} \cdots x_{r-1-i}}
$$

and it is easy to see as before that this coincides as rational function with

$$
-\frac{y_{1} \cdots y_{i}}{x_{i+1} \cdots x_{r}}
$$

and, for that matter, with any $-y^{I} / x^{J}$ as in (iii), so we may denote it by

$$
-\left[y^{i} / / x^{r-i}\right] \text {. }
$$

It is also easy to see as before that this standard open coincides with the regularity domain of this function, so we denote it by $U\left(\left[y^{i} / / x^{r-i}\right]\right)$. Similarly, we get a rational function $\left[x^{r-i} / / y^{i}\right]$.

Now we can prove (iii). Given $z \in W^{r}$ such that $w_{r}(z)$ is close to $(p, \ldots, p)$, we may assume $z$ projects to

$$
z^{\prime} \in U\left(\left[y^{i} / / x^{r-1-i}\right],\left[x^{r-i} / / y^{i-1}\right]\right) \subset W^{r-1},
$$


and, in particular,

$$
\begin{aligned}
& \frac{y_{1} \cdots y_{i} y_{r}}{x_{i+1} \cdots x_{r-1}}=\left[y^{i+1} / / x^{r-1-i}\right], \\
& \frac{x_{i} \cdots x_{r-1} x_{r}}{y_{1} \cdots y_{i-1}}=\left[x^{r-i+1} / / y^{i-1}\right]
\end{aligned}
$$

is regular at $z$. As we have seen, either

$$
\left[y^{i} / / x^{r-i}\right] \text { or }\left[x^{r-i} / / y^{i}\right]
$$

are regular at $z$. Therefore, either

$$
z \in U\left(\left[y^{i} / / x^{r-i}\right],\left[x^{r-i+1} / / y^{i-1}\right]\right)
$$

or

$$
z \in U\left(\left[x^{r-i} / / y^{i}\right],\left[y^{i+1} / / x^{r-1-i}\right]\right) .
$$

Thus (iii) is proved.

We will now prove (iv), which of course implies (ii) (our purpose in stating (ii) separately was rhetorical). To begin with, note that the question is local (on $W^{r}$, a fortiori on $X / B$ ) so we may assume $X / B$ is the versal family $x y=t$ over $B=\mathbb{A}^{1}$ (actually we just need that $B$ is integral). Now note the following claim.

Claim . (a) Over $U\left(\left[y^{i} / / x^{r-i}\right]\right), P_{i}^{r}$ is divisible by $x_{i+1} \cdots x_{r}$.

(b) Over $U\left(\left[x^{r-i} / / y^{i}\right]\right), P_{i}^{r}$ is divisible by $y_{1} \cdots y_{i}$.

Proof. We prove (a) as the proof of (b) is similar. Now $P_{i}^{r}$ (a polynomial in $x, y$ subject to the relation $\left.x y=x_{1} y_{1}\right)$ is a sum of terms of the form

$$
M_{i-a}(y) M_{r-i-b}(x) y^{a} x^{b}
$$

where the $M$ are monomials in distinct variables $y_{1}, \ldots, y_{i}, x_{i+1}, \ldots, x_{r}$ of the indicated degrees. If $a \leqslant b$, we use the relations

$$
x y=x_{j} y_{j}, \quad \forall j
$$

to rewrite this term as

$$
x^{b-a} y_{1} \cdots y_{i} M_{r-i-b+a}(x),
$$

which is clearly divisible as claimed. If $b \leqslant a$ this term can be rewritten as

$$
y^{a-b} M_{i-a+b}(y) x_{i+1} \cdots x_{r},
$$

which is even more clearly divisible as claimed. This proves the claim.

Note that the above calculation shows $P_{i}^{r} / x_{i+1} \cdots x_{r}$ and $P_{i-1}^{r} / y_{1} \cdots y_{i-1}$ can be written respectively as

$$
\begin{gathered}
y^{i}+f_{1}(x)+f_{2}(y), \\
x^{r+1-i}+g_{1}(x)+g_{2}(y)
\end{gathered}
$$

where $f_{1}, f_{2}$ have degree $\leqslant r-i$ and $g_{1}, g_{2}$ have degree $\leqslant i-1$, and all have regular functions as coefficients. Following the proof of [Ran05c, Theorem 2], we see that for $J_{r}$ as defined to yield a morphism

$$
J_{r}: W^{r}(X / B) \rightarrow \operatorname{Hilb}^{r}(X / B)
$$

is equivalent to certain identities among the coefficients of the $f_{i}$ and $g_{j}$. Since $J_{r}$ clearly coincides generically with the evident rational map, these identities hold generically, and hence they 


\section{Z. RAN}

hold period, so $J_{r}$ is indeed a lifting of the evident rational map as a morphism to Hilb. This completes the proof of (iv). Finally, in light of the fact that

$$
\frac{P_{i-1}^{r}\left(x_{r+1}, y_{r+1}\right)}{y_{1} \cdots y_{i-1}} / \frac{P_{i}^{r}\left(x_{r+1}, y_{r+1}\right)}{x_{i+1} \cdots x_{r}}=\left[x^{r-i+1} / / y^{i}\right],
$$

part (v) follows directly from (iv) and the definition of blow-up (the two opens in question are the standard affine opens of $\mathbb{P}^{1}$ over $U\left(\left[y^{i} / / x^{r-i}\right],\left[x^{r-i+1} / / y^{i-1}\right]\right)$. This completes the proof of Theorem 2.1.

A posteriori, we can identify $W^{r}(X / B)$ with the flag-Hilbert scheme studied in [Ran05c]: recall that the flag-Hilbert scheme $\mathrm{fHilb}_{m}(X / B)$ parametrizes $\mathcal{O}_{B}$-chains of ideals

$$
I_{m}<\cdots<I_{1}<\mathcal{O}_{X}
$$

where $\mathcal{O}_{X} / I_{j}$ is $\mathcal{O}_{B}$-locally free of length $j$. Note that the ideal

$$
\mathfrak{a}_{j}=\operatorname{Ann}\left(I_{j-1} / I_{j}\right)
$$

is $\mathcal{O}_{B}$-locally free of colength 1 , giving rise to a $B$-map fHilb $(X / B) \rightarrow X$. Putting these together, we get a map

$$
\mathrm{fHilb}_{m}(X / B) \rightarrow X^{m} / B .
$$

Note that the various maps $J_{j}$ together yield a morphism

$$
\eta_{r}: W^{r}(X / B) \rightarrow \mathrm{fHilb}_{r}(X / B),
$$

which evidently commutes with the natural maps of its source and target to $X^{r} / B$. We showed in [Ran05c] that any length- $m$ subscheme of a fibre of $X / B$ supported at a fibre node with local coordinates as above is either $Q_{i}^{m}=\left(x^{m-1+1}, y^{i}\right)$ or $I_{i}^{m}(a)=\left(y^{i}+a x^{m-i}\right), a \in \mathbb{C}^{*}$, and $a$ is part of a local coordinate system on $\operatorname{Hilb}_{m}(X / B)$.

Proposition 2.2. The map $\eta_{r}$ is an isomorphism.

Proof. The proof that follows uses the detailed, local-coordinate description of Hilb given in [Ran05c]. Clearly, both $W^{r}(X / B)$ and $\mathrm{fHilb}_{r}(X / B)$ are proper, even projective, over the fibre power $X^{r} / B$, and fHilb $r(X / B)$ is reduced by [Ran05c, Theorem 8]. Therefore, $\eta_{r}$ is scheme-theoretically surjective. To show that $\eta_{r}$ is an isomorphism, it suffices by an obvious induction to prove that its fibre over a flag supported at a point $p$ that is a relative node reduces (scheme-theoretically) to a point. By induction, we may assume $\eta_{r-1}$ is an isomorphism and, in particular, $\mathrm{fHilb}_{r}(X / B)$ is a subscheme of $W^{r-1} \times_{B} \operatorname{Hilb}_{r}(X / B)$. It then suffices to prove that a fibre $F$ of the natural map

$$
\zeta_{r}: W^{r} \rightarrow W^{r-1} \times_{B} \operatorname{Hilb}_{r}(X / B)
$$

is a point. Note that, because fHilb $_{r}(X / B)$ admits an $r$ th projection map to $X, F$ is contained in a fibre of the natural map

$$
b_{r}: W^{r} \rightarrow W^{r-1} \times_{B} X .
$$

Let $w \in W^{r}$ be 'supported' at a fibre node $p$ (i.e. $w_{r}(w)=(p, \ldots, p)$ or equivalently, $J_{r}(w)$ is supported at $p$ ) and write

$$
\zeta_{r}(w)=\left(w^{\prime}, z\right)
$$

Note also that, by construction, if

$$
w^{\prime} \in U\left(\left[y^{i} / / x^{r-1-i}\right],\left[x^{r-i} / / y^{i-1}\right]\right)
$$

then a fibre of the map $b_{r}$ is already coordinatized locally by either

$$
\left[y^{i} / / x^{r-i}\right] \text { or }\left[x^{r-i} / / y^{i}\right] \text {. }
$$




\section{GeOMETRY ON NODAL CURVES}

Assume first that $w \in U\left(\left[y^{i} / / x^{r-i}\right]\right)$. Then by [Ran05c, Theorem 2], and the computations in the proof of Theorem 2.1(iv) it is clear that $z$, the point in $\mathrm{Hilb}_{r}$ corresponding to $w$, is either

$$
I_{i}^{r}(a) \quad \text { with } a=\left[y^{i} / / x^{r-i}\right]
$$

if $a \neq 0$ (note that $a$ is the coefficient of $x^{r-i}$ in $P_{i}^{r} / x_{i+1} \cdots x_{r}$, and it has already been noted that, on $\operatorname{Hilb}_{r}, a$ is part of a coordinate system), or $Q_{i}^{r}$ if

$$
\left[y^{i} / / x^{r-i}\right]=\left[x^{r-i+1} / / y^{i-1}\right]=0 .
$$

Analogous conclusions hold if $w \in U\left(\left[x^{r-i} / / y^{i}\right]\right)$. In either case, the computations show that $\left[y^{i} / / x^{r-i}\right]$ or $\left[x^{r-i} / / y^{i}\right]$ can be read off from $z \in \mathrm{Hilb}_{r}$; consequently, $\zeta_{r}$ is scheme-theoretically injective and hence so is $\eta_{r}$.

Corollary 2.2.1. If $X / B$ is flat and $B$ is integral, $W^{r}(X / B) \rightarrow B$ is a flat, locally complete intersection morphism. In particular, if $B$ is Cohen-Macaulay or a locally complete intersection, so is $W^{r}(X / B)$.

Proof. It suffices to prove this locally on $X$. But locally, any $X / B$ is induced by base-change from the versal family $X_{0} / B_{0} \subset \mathbb{A}^{2} \times \mathbb{A}^{1} / \mathbb{A}^{1}$ given by $x y=t$. For this family, the analogous assertions for the flag Hilbert scheme were proven in [Ran05c, Theorem 8], and by base-change this implies the general case.

Unfortunately, $W^{r}$ is never smooth if $r \geqslant 3$, whenever $X / B$ has a singular fibre, but we still have the following proposition.

Proposition 2.3. If $B$ is smooth and $X / B$ is flat, then $W^{r}(X / B)$ is normal and Cohen-Macaulay.

Proof. We have essentially seen this already in [Ran05c] in the case of the flag-Hilbert scheme, but will give another proof for $W^{r}$. We first show inductively that $W^{r}$ is $R_{1}$. For $r=1$ this is clear (indeed $W^{1}=X$ is smooth or has at most rational double points). Inductively, if $W^{r-1}$ is $R_{1}$ then clearly so is $W^{r-1} \times_{B} X$. Moreover, the blow-up map

$$
b_{r}: W^{r} \rightarrow W^{r-1} \times_{B} X
$$

has at most $\mathbb{P}^{1}$ fibres and has those only over a codimension-3 locus. Hence $W^{r}$ is $R_{1}$.

Now one can see either by applying Corollary 2.2.1 above or imitating the foregoing proof that $W^{r}$ is Cohen-Macaulay and hence $\mathrm{S}_{2}$. Hence $W^{r}$ is normal.

\section{Enumerative results}

Now let

$$
\Gamma^{r+1} \subset W^{r} \times_{B} X
$$

be the 'tautological divisor' (i.e. the pullback of universal divisor over the Hilbert scheme via $J_{r}$ ). For any sheaf $L$ on $X$, set

$$
S^{r}(L)=p_{W^{r} *}\left(p_{X}^{*}(L) \otimes \mathcal{O}_{\Gamma^{r+1}}\right) .
$$

If $L$ is locally free, $S^{r}(L)$ is clearly locally free of $\operatorname{rank} r \cdot \operatorname{rk}(L)$, and we call it the $r$ th secant bundle associated to $L$. It was introduced in the smooth case by Schwarzenberger [Sch64]. Because $\Gamma^{r+1}$ is the pullback of the universal divisor on $\operatorname{Hilb}_{r}(X / B) \times_{B} X, S^{r}(L)$ is the pullback of the analogous bundle on $\operatorname{Hilb}_{r}(X / B)$. However, the recursive structure of $W^{r}$ makes $S^{r}(L)$ easier to compute with, as we proceed to show.

To start with, note trivially that, by flatness of $\Gamma^{r+1} / W^{r}, S^{r}$ is an exact functor from coherent (respectively locally free coherent) sheaves on $X$ to coherent (respectively locally free coherent) 


\section{Z. RAN}

sheaves on $W^{r}$. Also, if $V$ is a vector space and $\phi: V \otimes \mathcal{O}_{X} \rightarrow L$ is a map, $\phi$ lifts naturally to a map $V \otimes \mathcal{O}_{W^{r} \times_{B} X} \rightarrow p_{X}^{*}(L)$, whence a map

$$
\phi^{r}: V \otimes \mathcal{O}_{W^{r}} \rightarrow S^{r}(L),
$$

and clearly $\phi^{r}$ is surjective if $\phi$ is. Next, note that $W^{r}(X / B)$ is not symmetric with respect to permuting the factors, but still there are projections 'to the first $s$ factors', for all $s \leqslant r$ :

$$
\gamma^{r, s}: W^{r} \rightarrow W^{s}
$$

We also set

$$
\gamma^{r}=\gamma^{r, r-1}
$$

Also, denote by

$$
\Delta^{r} \subset W^{r}
$$

the exceptional divisor of $b_{r}$, i.e. $b_{r}^{*}\left(\Gamma^{r}\right)$, which is by definition of blow-up a Cartier divisor. As in Theorem 2.1, we have for any $r$ that $\Gamma^{r}$ splits up as

$$
\Gamma^{r}=\bigcup_{i=1}^{r-1} \Gamma_{i}^{r}
$$

with each $\Gamma_{i}^{r}$, being the graph of the $i$ th coordinate projection to $X$, isomorphic to $W^{r-1}$ and, in particular, reduced always and irreducible if $X$ is. Similarly, $\Delta^{r}$ splits

$$
\Delta^{r}=\bigcup_{i-1}^{r-1} \Delta_{i}^{r}
$$

with each $\Delta_{i}^{r}$ reduced and birational to $\Gamma_{i}^{r}$ (and, in general, a non-Cartier divisor on $W^{r}$ ).

Proposition 3.1. Let $p_{i}: W^{r}(X / B) \rightarrow X$ denote the $i$ th natural projection for $1 \leqslant i \leqslant r$. There is an exact sequence of vector bundles on $W^{r}(X / B)$

$$
0 \rightarrow p_{r}^{*}(L) \otimes \mathcal{O}\left(-\Delta^{r}\right) \rightarrow S^{r}(L) \rightarrow \gamma^{r *}\left(S^{r-1}(L)\right) \rightarrow 0 .
$$

Proof. There is clearly a natural surjection

$$
S^{r}(L) \rightarrow \gamma^{r *}\left(S^{r-1}(L)\right)
$$

whose kernel $K$ is locally free of $\operatorname{rank} \operatorname{rk}(L)$ and, moreover, admits a generically injective map

$$
k: K \rightarrow p_{r}^{*}(L) .
$$

Clearly, $k$ vanishes at each generic point of $\Delta^{r}$, and hence factors through $p_{r}^{*}(L) \otimes \mathcal{O}\left(-\Delta^{r}\right)$. It is easy to see that the factored map

$$
K \rightarrow p_{r}^{*}(L) \otimes \mathcal{O}\left(-\Delta^{r}\right)
$$

is an isomorphism in codimension 1 between locally free sheaves of the same rank, and hence is an isomorphism since $W^{r}$ is pure-dimensional.

Thus $S^{r}(L)$ has a natural filtration with quotients

$$
\left(\gamma^{r, s}\right)^{*}\left(p_{s}^{*}(L) \otimes \mathcal{O}\left(-\Delta^{s}\right)\right),
$$

and consequently we have the following corollary.

Corollary 3.2. The total Chern class $c\left(S^{r}(L)\right)$ satisfies

$$
c\left(S^{r}(L)\right)=\prod_{i=1}^{r} c\left(L_{i}\left(-\left(\gamma^{r, i}\right)^{*} \Delta^{i}\right)\right)
$$




\section{GeOMETRY ON NODAL CURVES}

where $L_{i}$ denotes (the class of) $p_{i}^{*}(L)$ and $\Delta^{1}=0$. In particular, if $L$ is invertible, we have

$$
c\left(S^{r}(L)\right)=\prod_{i=1}^{r}\left(1+L_{i}-\left(\gamma^{r, i}\right)^{*} \Delta^{i}\right) .
$$

After this was written, the author became aware of the work of Lehn [Leh99], which computes the Chern classes of the analogue of the $S^{r}(L)$ over Hilbert schemes of smooth surfaces, in terms of Nakajima's theory of the cohomology of these schemes. See [Ran05b] for a further discussion of the connection and for a verification that Corollary 3.2 is consistent with Lehn's formula.

In a nutshell, it is the possibility of results like the foregoing corollary that make the spaces $W^{r}$ amenable to enumerative computations. As a first application, we use this result to give a multiplepoint formula for maps to a Grassmannian. Let $X / B$ be as above and

$$
f: X \rightarrow G
$$

be a map to a Grassmannian $G=G(k, m+k)=G(k, V)$, with tautological sub- and quotient bundles $S_{G}, Q_{G}$, respectively. Set

$$
S_{X}=f^{*} S_{G}, \quad Q_{X}=f^{*} Q_{G} .
$$

Note that on $G \times W^{r}(X / B)$ we have a natural map

$$
\psi: p_{G}^{*}\left(S_{G}\right) \rightarrow p_{W}^{*} S^{r}\left(Q_{X}\right)
$$

which is the composite of the obvious inclusion $p_{G}^{*}\left(S_{G}\right) \subset V \otimes \mathcal{O}$ with the natural surjection $\phi^{r}: V \otimes \mathcal{O} \rightarrow p_{W}^{*} S^{r}\left(Q_{X}\right)$ deduced from the tautological quotient $\phi: V \otimes \mathcal{O} \rightarrow Q_{X}$. Another significant way to obtain $\psi$ is as follows. Consider the following diagram.

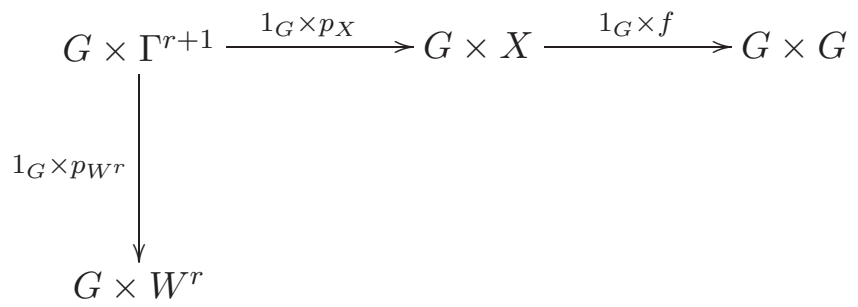

The equation $\psi_{0}$ of the diagonal in $G \times G$ is a section of $\operatorname{Hom}\left(p_{2}^{*} S_{G}, p_{1}^{*} S_{G}\right)$; by definition, we have

$$
p_{W^{*} *}\left(p_{X}^{*} f^{*}\left(Q_{G}\right)\right)=S^{r}\left(Q_{X}\right) .
$$

Then the image of $\psi_{0}$ under pulling back to $G \times \Gamma^{r+1}$ and pushing down to $G \times W^{r}$ is the map $\psi$. Thus, $\psi$ vanishes at a point $(w, g)$ if and only if all the elements of the subspace $g \subset V$ vanish in $Q_{X} \otimes \mathcal{O}_{J_{r}(w)}$; in other words, if and only if $f$ maps the scheme $J_{r}(w)$ corresponding to $w$ to the reduced point scheme $g$. We call the latter locus $M_{r}^{+}(f)$; by definition, it is a subscheme of $G \times W^{r}$. We may replace $M_{r}^{+}(f)$ by a subscheme of $W^{r}$ as follows. Define a bundle $S^{r, 1}(L)$, for any $L$, by the exact sequence

$$
0 \rightarrow S^{r, 1}(L) \rightarrow S^{r}(L) \stackrel{\alpha}{\rightarrow}\left(\gamma^{r, 1}\right)^{*}(L) \rightarrow 0 .
$$

Then clearly the zero-scheme of the composite map

$$
\alpha \circ \psi: p_{G}^{*}\left(S_{G}\right) \rightarrow p_{W}^{*}\left(\gamma^{r, 1}\right)^{*}\left(Q_{X}\right)
$$

is just the graph $R$ of the composite

$$
f \circ p_{1}: W^{r} \rightarrow G
$$

Thus, $M_{r}^{+}(f)$ is a subscheme of $R$ and, as such, it is the zero-scheme of a map $p_{G}^{*}\left(S_{G}\right) \rightarrow p_{W}^{*} S^{r, 1}(Q)$. Identifying $R$ with $W^{r}$ by projection, we see that $M_{r}^{+}(f)$ projects isomorphically to its image $M_{r}(f)$ 


\section{Z. RAN}

in $W^{r}$ and $M_{r}(f)$ is the zero-scheme of an analogous map

$$
p_{1}^{*}\left(S_{X}\right) \rightarrow S^{r, 1}\left(Q_{X}\right) .
$$

Then we have shown the following.

Theorem 3.3. In the above situation, $M_{r}^{+}(f)$ and $M_{r}(f)$ have a natural scheme structure as zeroscheme of vector bundle maps. When $M_{r}^{+}(f)$ and $M_{r}(f)$ have their expected codimension, i.e.

$$
\operatorname{codim}\left(M_{r}^{+}(f), W^{r} \times G\right)=r k m,
$$

or equivalently

$$
\operatorname{codim}\left(M_{r}(f), W^{r}\right)=(r-1) k m,
$$

then the cohomology (or rational equivalence) class of $M_{r}(f)$ on $W$ is

$$
\left[M_{r}(f)\right]=c_{(r-1) k m}\left(p_{1}^{*}\left(S_{X}^{*}\right) \otimes S^{r, 1}\left(Q_{X}\right)\right) .
$$

In the case when $G=\mathbb{P}^{m}=G(m, m+1)$, formula (3.5) simplifies somewhat. Let us write

$$
L_{i}=\left(f \circ p_{i}\right)^{*}\left(\mathcal{O}_{G}(1)\right), \quad M_{1}=\left(f \circ p_{1}\right)^{*}\left(S_{X}\right) .
$$

Then we get

$$
\left[M_{r}(f)\right]=\prod_{i=2}^{r} c_{m}\left(M_{1}^{*} \otimes\left(L_{i}-\left(\gamma^{r, i}\right)^{*}\left(\Delta^{i}\right)\right)\right)=\prod_{i=2}^{r}\left(\sum_{j=0}^{m} L_{1}^{j}\left(L_{i}-\left(\gamma^{r, i}\right)^{*}\left(\Delta^{i}\right)\right)^{m-j}\right) .
$$

Now consider the case $m=2, r=3$. Then the right-hand side of (3.5) yields

$$
\left(L_{1}^{2}+L_{1}\left(L_{2}-\Delta^{2}\right)+\left(L_{2}-\Delta^{2}\right)^{2}\right)\left(L_{1}^{2}+L_{1}\left(L_{3}-\Delta^{3}\right)+\left(L_{3}-\Delta^{3}\right)^{2}\right) .
$$

We want to compute the image of this on $X=W^{1}$. To this end we must first compute the image on the second factor on $W^{2}$ via $\gamma^{3,2}$. This computation follows formally from the following lemma.

Lemma 3.4. Set

$$
\begin{gathered}
f_{0}^{i}=\text { fibre of } W^{i}(X / B) \text { over } 0 \in B, \\
d=\operatorname{deg}\left(f_{0}^{1}\right)=f_{0}^{1} \cdot L, \quad b=\pi_{*} L^{2} .
\end{gathered}
$$

Then we have

(i) $\gamma_{*}^{3,2}\left(L_{1}^{2}\right)=0$,

(ii) $\gamma_{*}^{3,2}\left(L_{1} L_{3}\right)=d L_{1}$,

(iii) $\gamma_{*}^{3,2}\left(L_{1} \Delta^{3}\right)=2 L_{1}$,

(iv) $\gamma_{*}^{3,2}\left(L_{3}^{2}\right)=\pi_{2}^{*}(b)$,

(v) $\gamma_{*}^{3,2}\left(L_{3} \Delta^{3}\right)=L_{1}+L_{2}$,

(vi) $\gamma_{*}^{3,2}\left(\left(\Delta^{3}\right)^{2}\right)=-K_{1}-K_{2}+2 \Delta^{2}$,

where $K_{i}=p_{i}^{*}\left(K_{X / B}\right)$.

Proof. Assertions (i), (ii) and (iv) are obvious. Assertions (iii) and (v) are immediate from the fact that $\Delta^{3}$ has two components mapping birationally to $W^{2}$. To prove (vi) we may work off the (codimension-2) exceptional locus of the natural birational map

$$
W^{2} \rightarrow X^{2} / B
$$

and its inverse image in $W^{3}$; on this open set, $\Delta^{3}$ consists of two components $\Delta_{1}^{3}, \Delta_{2}^{3}$, each a pullback of the diagonal via the $p_{13}, p_{23}$ projections, which meet in a locus projecting isomorphically to $\Delta^{2} \subset W^{2}$. 


\section{GeOMETRY ON NODAL CURVES}

Remark 3.4.1. Actually, analogues of formulae (i)-(vi) hold for any $\gamma^{r, r-1}, r \geqslant 3$, in place of $\gamma^{3,2}$, where the analogue of (vi) is

$$
\left(\gamma^{r, r-1}\right)_{*}\left(\left(\Delta^{r}\right)^{2}\right)=-\sum_{i=1}^{r-1} K_{i}+2 \sum_{i=2}^{r-1}\left(\gamma^{r-1, i}\right)^{*}\left(\Delta^{i}\right)
$$

where we set $\gamma^{r-1, r-1}$ equal to the identity. All of these formulae are but the tip of a sizable iceberg, that is explored more deeply in [Ran04b].

Given the formulae (i)-(vi) in Lemma 3.4, an elementary formal calculation yields

$$
\gamma_{*}^{3,2}\left(\left(L_{1}^{2}+L_{1}\left(L_{3}-\Delta^{3}\right)+\left(L_{3}-\Delta^{3}\right)^{2}\right)\right)=(d-4) L_{1}-2 L_{2}-K_{1}-K_{2}+2 \Delta^{2}+\pi_{2}^{*}(b) .
$$

Therefore, by the projection formula the image of (3.7) via $\gamma^{3,2}$ is

$$
\left(L_{1}^{2}+L_{1}\left(L_{2}-\Delta^{2}\right)+\left(L_{2}-\Delta^{2}\right)^{2}\right) \cdot\left((d-4) L_{1}-2 L_{2}-K_{1}-K_{2}+2 \Delta^{2}+\pi_{2}^{*}(b)\right) .
$$

In the computation of the product in (3.8) and its image in $X$, the main point is the following. Observe that the normal bundle to $\Delta^{2}$ in $W^{2}$ is just $-b_{2}^{*} K_{1}+E$ where

$$
b_{2}: W^{2} \rightarrow X^{2} / B
$$

is the natural blow-up map and $E$ its exceptional locus, which is a divisor on $\Delta^{2}$. Indeed, the restriction of $b_{2}$ over the diagonal

$$
\Delta_{X}=X \subset X^{2} / B
$$

is just the blow-up of the critical locus of $\pi$, which we denote by $\sigma$ (cf. [Ran04a]). Therefore,

$$
\begin{gathered}
\gamma_{*}^{2,1}\left(\Delta^{2}\right)^{2}=-K, \\
\gamma_{*}^{2,1}\left(\Delta^{2}\right)^{3}=\gamma_{*}^{2,1}(K-E)^{2}=K^{2}-\sigma .
\end{gathered}
$$

From these facts the computation of (3.8) and its image in $X$ are routine.

Now by construction $M_{3}(f)$ parametrizes filtered length-3 schemes contained in fibres of $f$, and the filtration induces an ordering on the support. Therefore, the image on $X$ of $M_{3}(f)$ is geometrically twice the locus of points contained in a relative triple point of $f$, while the image on $B$ of the same is $6=3$ ! times the locus of fibres containing a triple point. Thus writing out the product yields the following result. As a matter of terminology, we will say that a locus $Z$ has 'virtual class $z$ ' if whenever $Z$ has its expected dimension, its cohomology or rational equivalence class is given by $z$.

TheOREM 3.5. Let $\pi: X \rightarrow B$ be a family of connected nodal curves of arithmetic genus $g$ and $f: X \rightarrow \mathbb{P}^{2}$ a morphism. Then the virtual class on $X$ of the locus of points contained in a relative triple point of $f$ is

$$
N_{3, X}(f)=\frac{1}{2}\left(\left(3 d^{2}-18 d+24+6 g-6\right) L^{2}+(18-3 d) K L+4 K^{2}-2 \sigma\right) ;
$$

the locus in $B$ of fibres containing a relative triple point of $f$ has virtual class

$$
N_{3, B}(f)=\frac{1}{3} \pi_{*}\left(N_{3, X}(f)\right) .
$$

Here $L=f^{*} \mathcal{O}(1), K=\omega_{X / B}, d=\operatorname{deg}\left(f\left(\pi^{-1}(\mathrm{pt}).\right)\right)$ and $\sigma$ is the class of critical locus of $f$.

Let us finally specialize to the case where $X / B$ is the normalization of the pencil (i.e. oneparameter family) of rational curves in $\mathbb{P}^{2}$ through $3 d-2$ assigned generic points. In this case all the ingredients of (3.9) have been computed recursively before, e.g. in [Ran99a, Ran99b, Ran01a, Ran01b]; the results needed from these papers are summarized in [Ran05a]. We have $L^{2}=N_{d}$, the 


\section{Z. RAN}

number of rational curves of degree $d$ through $3 d-1$ generic points, and also

$$
K=-2 s_{1}-m_{d} f_{0}^{1}+R_{1}
$$

(cf. [Ran05a, (11)]), where $s_{1}$ is a section of $X / B$ contracted by $f$ to a point (viz. one of the base points of the pencil), $R_{1}$ is the sum of all fibre components disjoint from $s_{1}$ and $m_{d}=-s_{1}^{2}$ is given by $[$ Ran05a, (5)]. Hence

$$
L . K=-d m_{d}+L \cdot R_{1}, K^{2}=-\sigma
$$

(cf. [Ran05a, (14),(15)]) and $\sigma$ coincides with the number of reducible fibres of $X / B$, also recursively computed. We conclude with the following corollary.

Corollary 3.6. The number of rational curves of degree $d$ in $\mathbb{P}^{2}$ having a triple point and going through $3 d-2$ generic points is (cf. [Ran04a])

$$
N_{d, 3}=\frac{1}{2}\left(\left(d^{2}-6 d+10\right) N_{d}+(d-6)\left(d m_{d}-L \cdot R_{1}\right)-2 \sigma\right) .
$$

Example 3.6.1. $N_{4,3}=60$, a number first computed by Zeuthen and rederived with modern methods by Kleiman and Piene [KP99]. ${ }^{1}$ See [KP99, Ran04a] for some similar examples.

When $f$ is a map to $\mathbb{P}^{m}$, one is interested classically not only in the relative multiple-point loci of $f$ but also in its relative multisecant loci, that is the locus of length- $r$ subschemes of fibres whose $f$-image is contained in a linear $\mathbb{P}^{k}$. This locus can be enumerated by the above results as the $r$-fold locus of the natural projection

$$
I_{X} \rightarrow \mathbb{G}(k, m):=G
$$

where $I_{X}$ is the incidence variety, i.e.

$$
I_{X}=\{(x, L): f(x) \in L\} \subset X \times G .
$$

However, it is simpler and more direct to enumerate this locus as follows. Set

$$
\operatorname{Sec}_{k}^{r}(f)=\{(z, L): f(\operatorname{sch}(z)) \subset L \text { as schemes }\} \subset W^{r}(X / B) \times G
$$

where $\operatorname{sch}(z)$ is the subscheme of $X$ corresponding to $z$. Clearly $\operatorname{Sec}_{k}^{r}(f)$ is just the zero-scheme of the natural map

$$
p_{2}^{*}\left(Q_{G}^{*}\right) \rightarrow S^{r}(L)
$$

where $Q_{G}$ is the tautological quotient bundle (of rank $(m-k)$ ) on $G$ and $L=f^{*}(\mathcal{O}(1)$ ). Thus we conclude the following.

Theorem 3.7. For a family of nodal curves $X / B$ and a morphism

$$
f: X \rightarrow \mathbb{P}^{m},
$$

the virtual locus on $W^{r}(X / B) \times G$ of relatively $r$-secant $k$-planes to $X / B$ in $\mathbb{P}^{m}$ is given by

$$
\left[\operatorname{Sec}_{k}^{r}(f)\right]=c_{r(m-k)}\left(p_{1}^{*}\left(S^{r}(L)\right) \otimes p_{2}^{*}\left(Q_{G}\right)\right) .
$$

If the right-hand side of (3.12) is nonzero (respectively not representable by an effective cycle), then the locus of relative $r$-secant $k$-planes is nonempty (respectively of dimension larger than the expected, viz. $\operatorname{dim}(B)+r+(k+r+2)(m-k))$.

Note that the projection of $\operatorname{Sec}_{k}^{r}(f)$ to $W^{r}(X / B)$ coincides with the locus where the natural map

$$
H^{0}\left(\mathcal{O}_{\mathbb{P}} m(1)\right) \otimes \mathcal{O}_{W^{r}(X / B)} \rightarrow S^{r}(L)
$$

has rank at most $k+1$, and consequently it can be enumerated directly via Porteous' formula [F].

\footnotetext{
${ }^{1}$ I am grateful to Steve Kleiman for this reference.
} 


\section{GeOMETRY ON NODAL CURVES}

Now Theorem 3.3 above 'repackages' the multiple-point locus $M_{r}^{+}(f)$ in a certain way, and it is possible to repackage it differently; the alternative repackaging is useful, notably, for recursion, and also allows some more general 'pseudo Grassmannian' target spaces. We proceed to define these.

Let us say that a smooth $m$-dimensional variety $G$ is pseudo-Grassmannian (with bundle $E$ and section $\psi$, if these need be specified) if $E$ is a rank- $m$ vector bundle on $G \times G$ and the diagonal

$$
\Delta_{G} \subset G \times G
$$

is the zero-scheme of the section $\psi$ of $E$.

Examples.

(i) Clearly a Grassmannian is pseudo-Grassmannian, with

$$
E=p_{1}^{*} S^{*} \otimes p_{2}^{*} Q
$$

where $S$ and $Q$ are respectively the tautological sub- and quotient bundles.

(ii) Trivially, any curve is pseudo-Grassmannian.

(iii) Generally, a product of pseudo-Grassmannians is pseudo-Grassmannian, therefore any product of curves and Grassmannians is pseudo-Grassmannian. More generally, a fibre product of Grassmannian bundles over a pseudo-Grassmannian is pseudo-Grassmannian. ${ }^{2}$

Now suppose we have a morphism $f: X \rightarrow G$ to a pseudo-Grassmannian with bundle $E$ and section $\psi$. Then we get a diagram as in (3.4), and pulling back and pushing forward $E$, $\psi$ we get a bundle $E^{r}$ with section $\psi^{r}$ on $G \times W^{r}$, and we define $M_{r}^{+}(f)$ as the zero scheme of $\psi^{r}$. As in the proof of Proposition 3.1, we have an exact sequence

$$
0 \rightarrow\left(p_{G} \times\left(f \circ p_{r}\right)\right)^{*}(E)\left(-\Delta^{r}\right) \rightarrow E^{r} \rightarrow\left(\gamma^{r, r-1}\right)^{*} E^{r-1} \rightarrow 0 .
$$

Consequently, $M_{r}^{+}(f)$ is a subscheme of $\gamma^{r, r-1 *}\left(M_{r-1}^{+}(f)\right)$ and as such is a zero scheme of $\left(p_{G} \times(f \circ\right.$ $\left.\left.p_{r}\right)\right)^{*}(E)\left(-\Delta^{r}\right)$. As in the Grassmannian case, the fact that $M_{r}^{+}(f)$ is contained in $\left(\gamma^{r, 1}\right)^{*} M_{1}^{+}(f)$, where $M_{1}^{+}(f)$ is just the graph of $f$, shows that $M_{r}^{+}(f)$ is isomorphic to its image $M_{r}(f)$ on $W^{r}$ and that, as a subscheme of $M_{r-1}(f), M_{r}(f)$ is a zero-scheme of $\left(\left(f \circ p_{1}\right) \times\left(f \circ p_{r}\right)\right)^{*}(E)\left(-\Delta^{r}\right)$.

Theorem 3.3 BIS. In the situation of Theorem 3.3, assume only that $G$ is pseudo-Grassmannian with bundle $E$. Then:

(i) $M_{r}(f)$ is a zero-scheme on $W^{r}$ of

$$
\bigoplus_{i=2}^{r}\left(\left(f \circ p_{1}\right) \times\left(f \circ p_{i}\right)\right)^{*}(E)\left(-\left(\gamma^{r, i}\right)^{*} \Delta^{i}\right)
$$

(ii) if $B$ is irreducible, if $M_{r}(f)$ is locally defined by $(r-1) m$ equations on $W^{r}(X / B)$ so hence is purely at least $(\operatorname{dim}(B)+r-(r-1) m)$-dimensional and if equality holds, then

$$
\left[M_{r}(f)\right]=\prod_{i=2}^{r}\left(\left(f \circ p_{1}\right) \times\left(f \circ p_{i}\right)\right)^{*}\left(c_{m}\left(E\left(-\left(\gamma^{r, i}\right)^{*} \Delta^{i}\right)\right)\right) .
$$

Proof. (i) has been proved above. In (ii), the assertion about the number of equations is clear from the definition. As for the assertion about the cohomology class, working by induction on $r$, it is clear from the Fulton-MacPherson residual-intersection formula [F] provided that both $M_{r}$ and $M_{r-1}$ have their expected dimensions. In the general case, let $C_{1}, \ldots, C_{k}$ be the irreducible

\footnotetext{
${ }^{2}$ Curiously (?), the author is unable to exhibit a single smooth variety that is (provably) not pseudo-Grassmannian.
} 


\section{Z. RAN}

components of $M_{r-1}$. By Fulton's theory, there is a cycle $U_{i}$ of dimension $\operatorname{dim}(B)+r-1-(r-2) m$ on each $C_{i}$ such that

$$
\sum\left[U_{i}\right]=m_{r-1}
$$

Since $M_{r}$ is locally defined by $m$ equations over $\left(\gamma^{r}\right)^{-1}\left(M_{r-1}\right)$ but still has its expected dimension, it follows that the contribution of each oversize component $C_{i}$ to $M_{r}$ is empty, and in particular

$$
\left(\gamma^{r}\right)^{*}\left(U_{i}\right) \cdot \mu_{r}(f)=0 .
$$

Therefore, these oversize components contribute nothing to either $M_{r}$ or $m_{r}$, so (3.14) still holds.

Although in most classical applications the target is in fact pseudo-Grassmannian, it is worth noting that Theorem 3.3 bis can be extended to mappings where the target is an arbitrary smooth $m$-dimensional variety $Y$ embedded in a pseudo-Grassmannian $G$. Note that any projective variety admits such an embedding (e.g. with $G$ a projective space). Thus, let

$$
f: X \rightarrow Y
$$

be a mapping to a smooth variety and fix an embedding

$$
Y \subset G
$$

in a pseudo-Grassmannian with bundle $E$. Of course, the diagonal $\Delta_{Y} \subset Y \times Y$ is a zero-scheme, albeit not of the expected dimension, of $E \otimes \mathcal{O}_{Y \times Y}$. Let $f^{\prime}: X \rightarrow G$ be the induced map. We set

$$
M_{r}(f)=M_{r}\left(f^{\prime}\right) \text {. }
$$

In fact, we will show that $M_{r}(f)$ depends only on $f$ and not the embedding $Y \subset G$. As noted above, as a subscheme of $\left(\gamma^{r, r-1}\right)^{-1}\left(M_{r-1}(f)\right), M_{r}(f)$ is a zero-scheme of a section of $\left(\left(f^{\prime} \circ p_{1}\right) \times\left(f^{\prime} \circ\right.\right.$ $\left.\left.p_{r}\right)\right)^{*}(E)\left(-\Delta^{r}\right)$ induced by a section $\psi$ of $E$ whose zero scheme is $\Delta_{G}$. The same section yields a section of $\left(\left(f^{\prime} \circ p_{1}\right) \times\left(f^{\prime} \circ p_{r}\right)\right)^{*}(E)$, whose zero-scheme consists of $\Delta^{r}$ plus

$$
\left(\left(f \circ p_{1}\right) \times\left(f \circ p_{r}\right)\right)^{*}\left(\Delta_{G} \cap Y \times Y\right)=\left(\left(f \circ p_{1}\right) \times\left(f \circ p_{r}\right)\right)^{*}\left(\Delta_{Y}\right) .
$$

Put another way, $M_{r}(f)$ is the residual scheme to $\Delta^{r}$ in $\left(\left(f \circ p_{1}\right) \times\left(f \circ p_{r}\right)\right)^{*}\left(\Delta_{Y}\right)$. This first shows that $M_{r}(f)$ is independent of the choice of embedding $Y \subset G$. Next, it allows us to compute $M_{r}(f)$ using residual-intersection theory. To that end, set for $k \geqslant 2$,

$$
\mu_{k}(f)=\left(\left(f \circ p_{1}\right) \times\left(f \circ p_{k}\right)\right)^{*}\left(\Delta_{Y}\right)-\Delta^{k}\left\{\frac{\left(f \circ p_{1}\right)^{*}\left(c\left(T_{Y}\right)\right)}{1+\Delta^{k}}\right\}_{m-1} .
$$

Also let

$$
m_{r}(f)=\prod_{k=2}^{r}\left(\gamma^{r, k}\right)^{*}\left(\mu_{k}(f)\right)
$$

where

$$
\gamma^{r, k}: W^{r}(X / B) \rightarrow W^{k}(X / B)
$$

is the natural map. The residual intersection formula of [Ful84, §9.2] yields that, whenever $M_{r}(f)$ has the expected codimension, viz. $m$, in $\left(\gamma^{r, r-1}\right)^{-1}\left(M_{r-1}(f)\right)$, then its class is given by $\mu_{r}(f)$. Thus we have the following theorem.

Theorem 3.3 Ter. Let $X / B$ be a family of nodal curves and

$$
f: X \rightarrow Y
$$

a morphism to a smooth $m$-dimensional variety embeddable in a pseudo-Grassmannian $G$. Then:

(i) there is a natural scheme structure $M_{r}(f)$ on the locus in $W^{r}(X / B)$ of points whose associated scheme is mapped by $f$ to a reduced point; 


\section{GeOMETRY ON NODAL CURVES}

(ii) if $B$ is irreducible, then $M_{r}(f)$ is locally defined by $(r-1) m$ equations on $W^{r}(X / B)$ and hence is purely at least $(\operatorname{dim}(B)+r-(r-1) m)$-dimensional; if $M_{r}(f)$ is $(\operatorname{dim}(B)+r-(r-1) m)$ dimensional or empty, then

$$
\left[M_{r}(f)\right]=m_{r}(f)
$$

Proof. Part (i) has been proved above and the proof of (ii) is essentially identical to that of Theorem 3.3 bis (ii). Note that the local defining equations for $M_{r}(f)$ in $\left(\gamma^{r, r-1}\right)^{-1}\left(M_{r-1}(f)\right)$ arise by pulling back equations for $\Delta_{Y}$ (or what is the same, for $\Delta_{G}$ ) and factoring out the equation of $\Delta^{r}$.

Remark. An earlier version of this paper gave a more general version of Theorem 3.3 ter, not assuming any embedding of $Y$, and with a more complicated proof. The foregoing argument is due to the referee. The general idea of defining a multiple-point locus recursively as a residual scheme inside a diagonal pullback is old folklore. Having the secant bundles is what makes it work.

Corollary 3.8. In the situation of Theorem 3.3 ter, if $m_{r}(f) \neq 0$ then $M_{r}(f)$ is nonempty.

Consider the case $r=2, m=3$. Thus we have a family of nodal curves mapping to $Y$ and are enumerating the relative multiple points of their images in $Y$ (at least if we assume that a general fibre of $X / B$ is smooth and embeds in $Y$, and that every fibre maps in with degree 1 ). Then it is easy to see that $(3.13)$ or $(3.15)$ specializes to (writing $f_{i}=f \circ p_{i}$ )

$$
\left[M_{2}(f)\right]=\left(f_{1} \times f_{2}\right)^{*}\left(\Delta_{Y}\right)-\left(\left(\Delta^{2}\right)^{3}-\left(\Delta^{2}\right)^{2} f_{1}^{*} K_{Y}+\Delta^{2} f_{1}^{*} c_{2}(Y)\right) .
$$

By the calculations in [Ran04a], we have as in the proof of Corollary 3.6,

$$
\begin{gathered}
p_{1 *}\left(\Delta^{2}\right)^{3}=K^{2}-\sigma \\
p_{1 *}\left(\left(\Delta^{2}\right)^{2} f_{1}^{*} K_{Y}\right)=-K \cdot f^{*}\left(K_{Y}\right), \quad p_{1 *}\left(\Delta^{2} f_{1}^{*} c_{2}(Y)\right)=f^{*}\left(c_{2}(Y)\right),
\end{gathered}
$$

where as before $K=\omega_{X / B}$ and $\sigma$ is the critical locus of $\pi$. Thus we obtain the following corollary. Corollary 3.9. For a family of nodal curves $X / B$ mapping via $f$ to a smooth pseudo-Grassmannian 3-fold $Y$, the virtual locus on $X$ of relative double points of $f$ is

$$
\left[N_{2, X}(f)\right]=p_{1 *}\left(f_{1} \times f_{2}\right)^{*}\left(\Delta_{Y}\right)-\left(K^{2}-\sigma+K . f^{*} K_{Y}+f^{*} c_{2}(Y)\right) .
$$

The expression $p_{1 *}\left(f_{1} \times f_{2}\right)^{*}\left(\Delta_{Y}\right)$ (which is a 0 -cycle or just a number if $B$ is one-dimensional) may be evaluated in various ways. For example, working in singular cohomology over $\mathbb{C}$, let $\left(\alpha_{i}\right)$ be a homogeneous basis for the total cohomology $H^{*}(B)$ and let $\left(\alpha_{i}^{*}\right)$ be the dual basis. Then the class of the diagonal $\Delta_{B}$ in $B \times B$ is given by

$$
\left[\Delta_{B}\right]=\sum \alpha_{i} \otimes \alpha_{i}^{*}
$$

Setting

$$
\beta_{i}=f_{*}\left(\pi^{*}\left(\alpha_{i}\right)\right), \quad \beta_{i}^{*}=f_{*}\left(\pi^{*}\left(\alpha_{i}^{*}\right)\right)
$$

we have

$$
f_{*}\left(p_{1 *}\left(f_{1} \times f_{2}\right)^{*}\left(\Delta_{Y}\right)\right)=\sum \beta_{i} \cdot \beta_{i}^{*}
$$

(which coincides with $p_{1 *}\left(f_{1} \times f_{2}\right)^{*}\left(\Delta_{Y}\right)$ or $\left(f_{1} \times f_{2}\right)^{*}\left(\Delta_{Y}\right)$ when they are of top degree, i.e. numbers). Note also that when $B$ is a curve we have

$$
\left[\Delta_{B}\right] \equiv[B] \otimes[p t]+[p t] \otimes B \bmod H^{1} \otimes H^{1},
$$

so if $H^{3}(Y)=0$ then the first term in $(3.19)$ reduces to $2 f_{*}\left(\left[f_{0}\right]\right) \cdot f_{*}([X])$. Finally, note that one customarily denotes

Thus we have the following.

$$
\pi_{*}\left(K^{2}\right)=\kappa, \quad \pi_{*}(\sigma)=\delta
$$




\section{Z. RAN}

Corollary 3.10. In the above situation, suppose:

(i) a generic fibre of $X / B$ is smooth and embedded via $f$;

(ii) $\operatorname{dim}(B)=1$;

(iii) $H^{3}(Y)=0$.

Then the virtual number of relative double points of $f$ is given by

$$
n_{2}(f)=\frac{1}{2}\left(2 f_{*}\left(\left[f_{0}\right]\right) \cdot f_{*}([X])-\kappa+\delta-K . f^{*} K_{Y}-f^{*} c_{2}(Y)\right) .
$$

In particular, if $n_{2}(f) \neq 0$ then $f$ does not embed all fibres of $X / B$ and if $n_{2}(f)<0$ then $f$ has degree $>1$ on some fibre.

We note that, if $Y=\mathbb{P}^{3},(3.13)$ simplifies to

$$
n_{2}(f)=\frac{1}{2}\left(2 b d-6 b-4 L \cdot K-K^{2}\right)
$$

where as usual $L=f^{*} \mathcal{O}(1), d=L . \pi^{-1}\left(p t\right.$.) and $b=L^{2}$. This formula was first given in [CR94] where it was derived from a general double-point formula referred to Fulton's book [Ful84] (in fact, the book does not appear to contain such a formula explicitly in this generality, although it should be possible to derive one from the case treated there, due to the fact that any double point is automatically curvilinear). As shown in [CR94], Corollary 3.10 has the following geometric consequence.

COROLlary 3.11. There is no nontrivial family of nonsingular rational curves of degree $d \geqslant 3$ in $\mathbb{P}^{3}$ parametrized by an irreducible projective variety of positive dimension.

Proof. We reproduce the short argument from [CR94]. If the assertion fails, there is a family as above with $X / B$ a $\mathbb{P}^{1}$-bundle $\mathbb{P}(E)$, where $E$ is a rank-2 vector bundle over a smooth curve $B$ and $f$ restricted on each fibre is an embedding in $\mathbb{P}^{3}$, so that $n_{2}(f)=0$; moreover, $f(X)$ is twodimensional, so that $b=L^{2}>0$. With no loss of generality, one may assume $c_{1}(E)=0$ or -1 . Set $D=c_{1}\left(\mathcal{O}_{\mathbb{P}(E)}(1)\right)$. If $c_{1}(E)=0$, we have

$$
K=-2 D, K^{2}=0,
$$

and we can write

$$
L=d D+x F, F=\pi^{-1}(p t .), \quad x \in \mathbb{Z} .
$$

Since $b=L^{2}>0$, we have $x>0$, and hence

$$
L . K=-2 x<0 .
$$

Since $d \geqslant 3,(3.21 \mathrm{bis})$ yields a contradiction. If $c_{1}(E)=-1$, we can write, with similar notation,

$$
K=-2 D+F, D^{2}=1
$$

so

$$
K^{2}=0, L . K=-d-2 x<0,
$$

and therefore again (3.21bis) yields a contradiction.

As another special case of Corollary 3.10, we recover a result from [Ran04a]. We use the notation developed in [Ran05a]; in particular, $N_{d}^{\text {red }}(a$.) denotes the number of reducible rational curves of degree $d$ in $\mathbb{P}^{3}$ satisfying the incidence conditions indicated by (a.) and $m_{1}=-s_{1}^{2}$ where $s_{1}$ is the section of $X / B$ corresponding to an incident linear subspace of codimension $a_{1}$. Both these numbers are recursively computable. See [Ran05a] for more details.

COROLlary 3.12. With the notation of [Ran05a], the number of singular rational curves of degree $d$ through a generic points and $4 d-2 a-1$ generic lines in $\mathbb{P}^{3}$ is

$$
(d-2) N_{d}\left(3^{a} 2^{4 d-2 a}\right)+N_{d}^{\text {red }}\left(3^{a} 2^{4 d-2 a-1}\right)-2 d m_{1}\left(3^{a} 2^{4 d-2 a-1}\right)+2 L \cdot R_{1}
$$




\section{GeOMETRY ON NODAL CURVES}

if $a>0$ and

$$
(d-2) N_{d}\left(3^{a} 2^{4 d-2 a}\right)+N_{d}^{r e d}\left(3^{a} 2^{4 d-2 a-1}\right)-2 d m_{a+1}\left(3^{a} 2^{4 d-2 a-1}\right)+2 L \cdot R_{a+1}-4 N_{d}\left(3^{a+1} 2^{4 d-2 a-2}\right)
$$

if $4 d-2 a-1>0$.

Proof. We use Corollary 3.10 for the family $X / B\left(3^{a} 2^{4 d-2 a-1}\right)$. Then the right-hand side of (3.21bis) yields, with $(a)=.\left(3^{a} 2^{4 d-2 a-1}\right)$, that in the above notation

$$
n_{2}(f)=\frac{1}{2}\left(2 d N_{d}\left(3^{a} 2^{4 d-2 a}\right)-K^{2}+N_{d}^{\mathrm{red}}(a .)+4 L K-4 N_{d}\left(3^{a} 2^{4 d-2 a}\right)\right) .
$$

Using the formula [Ran05a, (14)] for $i=1$ and noting that $N_{d}(4, \ldots)=$,0 yields $(3.22)$, while the same formula with $i=a+1$ yields (3.23).

Remark 3.13. Theorem 3.3, as well as the other multiple-point results in this paper, admit straightforward generalizations to the relative case, where $Y$ is replaced by a smooth morphism

$$
\rho: Y \rightarrow B
$$

and $f$ is a $B$-morphism, i.e. the following diagram commutes.

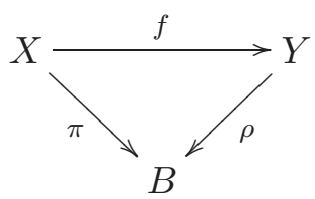

Note that the 'absolute' case discussed above becomes a special case of the relative case by replacing $Y$ by $Y \times B \rightarrow B$. In the relative case the factors $\mu_{k}(f)$ are replaced by

$$
\mu_{k}(f / B)=\left(f_{1} \times_{B} f_{k}\right)^{*}\left(\Delta_{Y / B}\right)-\Delta^{k}\left\{\frac{f_{1}^{*}\left(c\left(T_{Y / B}\right)\right)}{1+\Delta^{k}}\right\}_{m-1}
$$

where $\Delta_{Y / B}$ is the diagonal in $Y \times_{B} Y$ and $T_{Y / B}$ is the relative or vertical tangent bundle of $Y \rightarrow B$ (which coincides with the normal bundle of $\Delta_{Y / B}$ in $Y \times_{B} Y$ ) and $m$ is the relative dimension of $Y / B$. The analogue of Theorem 3.3 with its bis and ter (with (3.24) in place of (3.15)), as well as their consequences, hold. The proofs are the same, because for a $B$-map $f$, multiple-point loci involve only the 'vertical' coordinates of $Y$ over $B$.

Concluding remark 3.14. Hopefully, the enumerative results of this section provide sufficient motivation for wanting to determine completely the multiplicative structure of the subring of the Chow ring of $W^{r}$ generated by $\Delta^{2}, \ldots, \Delta^{r}$ together with the Chow ring of $X$. As remarked above, this indeed is the subject of [Ran04b].

\section{ACKNOWLEDGEMENT}

The author thanks the referee for his detailed, constructive suggestions which have improved the paper.

\section{REFERENCES}

Ang81 B. Angéniol, Familles de cycles algébriques - schéma de Chow, Lecture Notes in Mathematics, vol. 896 (Springer, Berlin-New York, 1981).

CR94 M. C. Chang and Z. Ran, Dimension of families of space curves, Compositio Math. 90 (1994), $53-57$.

Ful78 W. Fulton, A note on residual intersections and the double point formula, Acta Math. 140 (1978), 93-101. 


\section{Geometry on nodal CURVES}

Ful84 W. Fulton, Intersection theory, Ergeb. Math. Grenzgeb. (3), vol. 2 (Springer, Berlin, 1984).

Kle81 S. Kleiman, Multiple-point formulas I: Iteration, Acta Math. 147 (1981), 13-49.

Kol96 J. Kollár, Rational curves on algebraic varieties, Ergeb. Math. Grenzgeb. (3), vol. 32 (Springer, Berlin, 1996).

KP99 S. Kleiman and R. Piene, Enumerating singular curves on surfaces, in Algebraic geometry: Hirzebruch 70, Contemporary Mathematics, vol. 241 (American Mathematical Society, Providence, RI, 1999), 209-238; corrections and revision in math.AG/9903192.

Leh99 M. Lehn, Chern classes of tautological sheaves on Hilbert schemes of points on surfaces, Invent. Math. 136 (1999), 157-207.

Mac62 I. G. MacDonald, Symmetric products of an algebraic curve, Topology 1 (1962), 319-343.

Mat65 A. Mattuck, Secant bundles on symmetric products, Amer. J. Math. 81 (1965), 779-797.

Mum88 D. Mumford, The red book of varieties and schemes, Lecture Notes in Mathematics, vol. 1358 (Springer, Berlin, 1988).

Ran85 Z. Ran, Curvilinear enumerative geometry, Acta Math. 155 (1985), 81-101.

Ran99a Z. Ran, Bend, break and count, Israel J. Math. 111 (1999), 109-124.

Ran99b Z. Ran, Bend, break and count II, Math. Proc. Camb. Phil. Soc. 127 (1999), 7-12.

Ran01a Z. Ran, On the variety of rational space curve, Israel J. Math. 122 (2001), 359-370.

Ran01b Z. Ran, The degree of the divisor of jumping rational curves, Quart. J. Math. 52 (2001), 367-383.

Ran04a Z. Ran, Enumerative geometry of divisorial families of rational curves, Ann. Sc. Norm. Super. Pisa Cl. Sci. (5) 3 (2004), 67-85.

Ran04b Z. Ran, Geometry on nodal curves II: cycle map and intersection calculus, Preprint (2004), math.AG/0410120.

Ran05a Z. Ran, Rational curves in projective spaces, unpublished notes (2005). Available at: http://math.ucr.edu/ ziv/papers/ratcurv.pdf.

Ran05b Z. Ran, Cycle map on Hilbert schemes of nodal curves, in Proc. conf. algebraic geometry, Siena, 2004, ed. L. Chiantini (de Gruyter, Berlin, 2005), to appear.

Ran05c Z. Ran, A note on Hilbert schemes of nodal curves, J. Algebra (2005), to appear.

Sch64 R. L. E. Schwarzenberger, The secant bundle of a projective variety, Proc. London Math. Soc. (3) 14 (1964), 369-384.

Ziv Ran ziv@math.ucr.edu

Department of Mathematics, University of California, 900 Big Springs Drive, Riverside, CA 92521, USA 